\title{
Are 3C 249.1 and 3C 334 restarted quasars? (Research Note)
}

\begin{abstract}
A. Marecki
Toruń Centre for Astronomy, N. Copernicus University, 87-100 Toruń, Poland

e-mail: amr@astro.uni.torun.pl

Received 13 July 2012 / Accepted 23 August 2012

\section{ABSTRACT}

This Research Note follows up a Letter in which I posit that $\mathrm{J} 1211+743$ is a restarted radio source. This means that its structure, where the jet points to the relic lobe, is only apparently paradoxical. Here, I propose the same scenario and apply the same mathematical model to 3C 249.1 and 3C 334. The ultimate result of my investigation is that these two well-known radio-loud quasars can be understood best so far if it was assumed that they, too, had been restarted.
\end{abstract}

Key words. radio continuum: galaxies - galaxies: active

\section{Introduction}

In principle, the "twin exhaust" model of a classical radio source (Blandford \& Rees 1974) predicts full symmetry of its doubled structures, i.e. lobes and jets, but this is very often not the case in reality. The main cause of asymmetries is the source orientation leading to beaming and Doppler-boosting that explain the apparent one-sidedness of the jets very well. However, asymmetries of the lobes cannot be attributed to relativistic beaming because they are not moving with relativistic velocities (Longair \& Riley 1979). A good example of a class of object where asymmetries are conspicuous, but do not result from the orientation, are the so-called HYbrid MOrphology Radio Sources (HYMORS) (Gopal-Krishna \& Wiita 2000; Gawroński et al. 2006), i.e. sources each of whose sides are a different Fanaroff-Riley (FR) type (Fanaroff \& Riley 1974). Earlier, Gopal-Krishna et al. (1996) highlighted several radio-loud quasars in which the hotspot on the jetted side was barely detected. The authors coined the term "weak-headed quasars" for them and argued that the faintness of the nearer hotspot, i.e. the one on the jet side, stemmed from the differential light-travel time caused by the source orientation so that the near-side lobe was viewed at a later evolutionary stage as compared to the far-side lobe.

Marecki (2012, hereafter Paper I) independently used the same argument to show that in J1211+743 (4CT 74.17.01), a source which, at first sight, could be labelled a HYMORS, the asymmetric appearance of the lobes also resulted from differential light-travel time. Therefore, J1211+743 is a pseudoHYMORS, not a real one, whereas the correlation between the jet direction and the location of the relic lobe is only apparently paradoxical. The case of J1211+743 is important for yet another reason. It has been shown in Paper I that this object must have been restarted to appear the way it does. Consequently, not only the so-called double-double sources are those where activity restart surely takes place - see the review of Saikia \& Jamrozy (2009). It thus seems timely to search for other objects similar to J1211+743 and, if found, to check using the formalism developed in Paper I whether their observed geometry fits the activity re-ignition scenario combined with the differential light-travel time.

\section{In quest of $\mathrm{J} 1211+743$ analogues}

In the 607-MHz map of J1211+743 (Pirya et al. 2011), the tip of its one-sided jet is located close to the boundaries of its relic lobe. However, from the point of view of the scenario presented in Paper I, this circumstance is not at all essential for the model to remain valid. The exact location of the tip of the jet can be largely arbitrary within the lobe itself or its vicinity, although it obviously cannot coincide with the expected location of the hotspot, otherwise it would be hardly possible to prove that the relic had no hotspot. When looking for other objects analogous to $\mathrm{J} 1211+743$, therefore, the candidates only have to be tested against the following two criteria:

1. the source has one FR II-like lobe with a hotspot and one diffuse relic lobe without a hotspot;

2. the source has a one-sided jet and the relic is on the jet side.

I visually inspected the images of double-lobed radio sources published in Black et al. (1992), Lonsdale et al. (1993), Price et al. (1993), Bogers et al. (1994), Bridle et al. (1994), Law-Green et al. (1995), Neff et al. (1995), Reid et al. (1995, 1999), Fernini et al. (1997), Hardcastle et al. (1997), Harvanek \& Hardcastle (1998), Riley et al. (1999), Gilbert et al. (2004, hereafter G04), Fernini (2007), and Kharb et al. (2008) to find objects meeting these criteria. It turned out that only two sources 3C 249.1 and 3C 334 - clearly met them, so they are discussed in detail below. Additionally, I made an exception for $1317+520$ and $2209+152$, and despite not meeting the criteria by these objects, I consider them in Sect. 2.4.

\subsection{The case of $3 C 249.1$}

The object 3C 249.1 (PG 1100+772) is a $z=0.3115$ quasar whose radio structure is best shown in Figs. 5 and 6 of Lonsdale \& Morison (1983), Figs. 18-21 of Bridle et al. (1994), and Figs. 37-39 of G04. The western lobe is FR II type while the eastern one is devoid of a hotspot and remarkably featureless. This, together with a high value of the spectral index $\alpha \sim 0.9$ (G04), which is directly responsible for a complete absence of 
eastern lobe in the 8.4-GHz image of 3C 249.1 (Fernini 2007), combined with the diffuseness of that lobe, which is best seen in the 408-MHz image (Lonsdale \& Morison 1983), provide compelling evidence that the eastern lobe is a relic.

A number of explanations of the peculiar morphology of 3C 249.1 have been proposed in the literature - see G04 for references - including the "flip-flop" mechanism (Lonsdale \& Morison 1983). According to these authors, the jet was once directed to the east, and it inflated the eastern lobe. After a reversal, which is the essence of the "flip-flop" model, the jet was oriented westerly so that the western lobe developed. The subsequent reversal is reflected in the direction of the jet as currently perceived. Although the "flip-flop" mechanism is no longer used to interpret double-lobed radio sources, one element of that theory still remains viable: the jet is currently not energising the eastern lobe because these two components belong to different epochs and their alignment is a mere spatial coincidence. This assertion is a cornerstone of the scenario I propose for 3C 249.1, the same as the one devised for J1211+743 in Paper I.

My model posits that, instead of the reversals of the intrinsically one-sided jet, the activity of the nucleus is recurrent and both jets were not produced for some time - a quiescence period, $t_{\mathrm{q}}$. The transition of the nucleus to the quiescent mode combined with a short lifetime of the hotspot $\left(7 \times 10^{4} \mathrm{yr}\right.$, Kaiser et al. 2000) led to the swift disappearance of hotspots. However, if the source does not lie on the sky plane then the differential light-travel time makes it possible that for a limited period of time the relic is seen only on the near side. If the activity of the nucleus is reinstated within that time window then we observe a superposition of two effects: the lobes' decay asymmetry and the presence of apparently one-sided jet pointing to the relic lobe. It has been shown in Paper I that the above scenario requires fine-tuned timing, and the necessary formalism has been developed there. I apply it here to test whether the model is valid for $3 \mathrm{C} 249.1$. The results are given in Sect. 2.3.

\subsection{The case of $3 C 334$}

The object 3 C 334 is a $z=0.5551$ quasar whose radio structure is best shown in Figs. 24-26 of Bridle et al. (1994), and Figs. 52-54 of G04. The western lobe is FR II type while the eastern one is somewhat peculiar. In the 4.9-GHz VLA map (Bridle et al. 1994, Fig. 25a), this lobe, although quite diffuse in general, has three features denoted as "Q", "R", and "S". None of these three dominates to make it a good candidate for the actual hotspot. On the other hand, in the 5-GHz VLA image shown in Fig. 54 in G04, the eastern lobe is rather featureless. G04 claim that there is a "clear trail of emission" made of components "S4" and "S5" that provides a connection from feature "S3" in the jet all the way to feature "S6" in the lobe. It could be, however, asserted equally well that there is a clear gap in emission between components "S4" and "S5" - see the lower panel of Fig. 54 - and the lobe itself is isolated. Therefore, this image provides substantial ground for suspecting that the eastern lobe of 3C 334 is in fact a relic that is currently not fuelled by the jet that terminates with component "S3" at the lobe's outskirts.

I thus propose the following interpretation of 3C 334. The activity of its nucleus ceased at some point in the past. This led to the cut-off of the stream of relativistic plasma to both lobes we still observe. The western lobe is farther from us. That's why we perceive it as a being of FR II type, while the eastern lobe is seen as a relic of a former FR II-type lobe. After a period of quiescence $t_{\mathrm{q}}$, the activity was renewed and the jet is a conspicuous signature of that. However, the central engine of 3C 334 was repositioned during the restart so now the jet misses the eastern lobe slightly and is not connected to it; the alleged bridge made of components "S4" and "S5" is an illusion caused by the bend of the jet. (The bend of the jet is likely to be forced by the pressure gradient on the boundary of the cocoon of the radio source.)

Based on the above scenario, application of the model developed in Paper I to 3C 334 seems to be justified. However, because of the misalignment between the straight section of the jet and the line connecting the core and the position of the tip of the relic lobe, the meaning of $d$ (see Fig. 1 of Paper I) has changed it is now the length of the projection of the jet onto that line. Consequently, $\beta_{\text {adv }}$ is not the actual jet advance speed but the speed of the tip of the jet projection, so the true $\beta_{\text {adv }}$ is somewhat higher than the values of projected $\beta_{\text {adv }}$ used in the model.

\subsection{Application of the model to $3 C 249.1$ and $3 C 334$}

In Paper I, the constraints on the length of the quiescence period $t_{\mathrm{q}}$ were analysed, and it was shown that $t_{\mathrm{q}}$ must stay within certain limits for the model to be valid. To calculate the lower and the upper limits to $t_{\mathrm{q}}$, one has to use Eqs. (2) and (4) (Paper I), respectively. They require several parameters: $l_{1}, l_{2}$, $d$ - see Fig. 1 of Paper I for the explanation of their meaning have to be extracted from the images of the sources; $\beta_{\text {adv }}$ and $\beta_{\text {jet }}$, which are common for 3C 249.1 and 3C 334, are taken from the literature; and $\theta$ (see Fig. 1 of Paper I) is a free parameter. Arshakian \& Longair (2004) calculated jet speeds for a number of double sources including 3C 249.1 and 3C 334 and they obtained $\beta_{\text {jet }} \approx 0.8$ for them. It is adopted here. As for $\beta_{\text {adv }}$, I use four values from the range $0.2 \leq \beta_{\mathrm{adv}} \leq 0.35$ that is similar to the one found for B $1834+620$ by Schoenmakers et al. (2000).

On the basis of the map of 3C 249.1 in G04 and using the standard cosmological parameters, I adopted the following parameters for this source: $l_{1} \sin \theta=88 \mathrm{kpc}, l_{2} \sin \theta=128 \mathrm{kpc}$, $d \sin \theta=41 \mathrm{kpc}$. Since one of the lobes is diffuse, estimating the size of the whole source reliably is hardly possible. What I did was simply to measure it based on the range of the lowest contour in the published figure. After substituting them to Eq. (2) of Paper I, I found that it was fulfilled for any value of $\theta$ if $\beta_{\mathrm{adv}}=0.2$, for $\theta<78.8$ if $\beta_{\mathrm{adv}}=0.25$, for $\theta<61^{\circ} .3$ if $\beta_{\mathrm{adv}}=0.3$, and for $\theta<47.8$ if $\beta_{\mathrm{adv}}=0.35$. Given that 3C 249.1 is a quasar and that according to Barthel (1989) $\theta<44^{\circ} .4$ for quasars, the above constraints on $\theta$ are not critical.

On the basis of the map of 3C 334 in G04 and using the standard cosmological parameters, I adopted the following parameters for this source: $l_{1} \sin \theta=206 \mathrm{kpc}, l_{2} \sin \theta=156 \mathrm{kpc}$, $d \sin \theta=106 \mathrm{kpc}$. As in the case of $3 \mathrm{C} 249.1$, I measured the source's size based on the range of the lowest contour in the published figure. After substituting them to Eq. (2) of Paper I, I found that it was fulfilled for any value of $\theta$ if $0.2 \leq \beta_{\text {adv }} \leq 0.35$.

Substitution of the parameters for 3C 249.1 and 3C 334 to Eq. (4) of Paper I yielded the upper limits to the length of the quiescent period for the respective sources. They are given in Table 1 for a wide range of values of $\theta$. The lack of a number at the crossing of a given row and column of Table 1 means that the respective combination of parameters is not allowed if the scenario is to be viable. On the other hand, following the argument in Paper I, the existence of upper limits for the remaining combinations of parameters proves that the model is plausible.

\subsection{The cases of $2209+152$ and $1317+520$}

The object $2209+152$ is a $z=1.502$ quasar whose entire radio structure is best shown in Fig. 159 of Lonsdale et al. (1993). It 
Table 1. Upper limits to the length of $t_{\mathrm{q}}$ in $3 \mathrm{C} 249.1$ and 3C 334 [Myr].

\begin{tabular}{l|cccc|cccc}
\hline \hline & \multicolumn{5}{|c|}{3 C 249.1 } & \multicolumn{4}{c}{3 C 334 } \\
$\theta$ & \multicolumn{4}{|c|}{$\beta_{\text {adv }}$} \\
{$\left[{ }^{\circ}\right]$} & 0.2 & 0.25 & 0.3 & 0.35 & 0.2 & 0.25 & 0.3 & 0.35 \\
\hline 10 & 0.60 & 1.37 & 1.88 & 2.25 & 0.65 & 2.64 & 3.97 & 4.92 \\
20 & 0.25 & 0.64 & 0.90 & 1.09 & 0.20 & 1.21 & 1.88 & 2.36 \\
30 & 0.11 & 0.38 & 0.55 & 0.68 & - & 0.68 & 1.14 & 1.47 \\
40 & 0.02 & 0.23 & 0.37 & 0.46 & - & 0.37 & 0.73 & 0.98 \\
50 & - & 0.12 & 0.24 & 0.32 & - & 0.14 & 0.45 & 0.66 \\
60 & - & 0.04 & 0.14 & 0.22 & - & - & 0.23 & 0.42 \\
70 & - & - & 0.06 & 0.13 & - & - & 0.04 & 0.21 \\
80 & - & - & - & 0.05 & - & - & - & 0.03 \\
\hline
\end{tabular}

consists of a core, a curved jet and two lobes, both relics. It can be speculated that $2209+152$ is restarted, i.e. the lobes were inflated in the previous active period but now are fading out due to the lack of fuelling, while the jet is a signature of the current active phase. The radio source $2209+152$ is not featured by the lobe asymmetry that could give us a clue to its orientation caused by differential light-travel time. Nevertheless, owing to the presence of a conspicuous Laing-Garrington effect (Laing 1988; Garrington et al. 1988) in this source (Garrington et al. 1991, Fig. A25) it is confirmed that the source does not lie in the sky plane, the eastern, i.e. the jet-side, lobe being nearer the observer. It follows that $2209+152$ could possibly meet the criteria listed at the beginning of this section. An explanation for why it does not meet them anyway is quite straightforward: the actual quiescence period of its nucleus must have exceeded the upper limit calculated from the model for given parameters. As a result, the information that the far-side lobe had started to decay reached the location of the observer too early relative to the development of the jet and now we perceive that lobe as a relic. In other words, wavefront " 1 " passed the observer earlier than wavefront "5" - see Fig. 1 in Paper I.

The above speculation can be extended. If the length of the quiescence period goes beyond the upper limit given by the model even further than in the case of $2209+152$, then the nearside lobe vanishes, while the far-side lobe remains barely observable. A $z=1.061$ quasar 1317+520 imaged at several frequencies by Reid et al. (1995, Fig. 28), but see also Jorstad \& Marscher (2008, Fig. 2), is perhaps a good specimen to illustrate such a situation: there is no trace of the lobe on the jetted side, whereas the lobe on the opposite side is clearly a relic, because it is devoid of a hotspot and very diffuse.

\section{Concluding remarks}

Cessation of activity in galactic nuclei, possibly followed by its restart, is a phenomenon that can manifest itself in a number of ways, the existence of double-double radio sources being perhaps the most spectacular as shown in the review of Saikia \& Jamrozy (2009). A restarted radio source need not be symmetric, though. For example, Marecki \& Swoboda (2011) highlighted a small group of galaxies where the production of the jet had stopped, leading to the decay of the radio lobes. Owing to the light-travel time difference between the lobes, that decay is perceived as asymmetric. In Paper I, the effect of differential light-travel time was analysed quantitatively also taking the activity re-ignition into account. The analysis carried out there proved that the scenario suggested for the sources with asymmetric lobes and the jet pointing to the relic was plausible for at least one source: J1211+743.

Here, I show that two well-known quasars, 3C 249.1 and 3C 334, can also be interpreted this way and that they have been restarted as well. The key parameter of my model is the upper limit to duration of the quiescent period between the two active periods, $t_{\mathrm{q}}$. Possible values of the upper limits to $t_{\mathrm{q}}$ for 3C 249.1 and 3C 334 have been displayed in Table 1. They are lower that their counterparts for J1211+743 shown in Table 1 of Paper I. This can be attributed to smaller sizes of 3C 249.1 and 3C 334 as compared to $\mathrm{J} 1211+743$. Also, it is interesting to note that all the values of the upper limits to $t_{\mathrm{q}}$ shown here are of the same order of magnitude as those obtained for J0041+3224 and J1835+6204 by Konar et al. (2012) using a completely different approach.

Acknowledgements. This research has made use of the NASA/IPAC Extragalactic Database (NED), which is operated by the Jet Propulsion Laboratory, California Institute of Technology, under contract with the National Aeronautics and Space Administration.

\section{References}

Arshakian, T. G., \& Longair, M. S. 2004, MNRAS, 351, 727

Barthel, P. D. 1989, ApJ, 336, 606

Black, A. R. S., Baum, S. A., Leahy, J. P., et al. 1992, MNRAS, 256, 186 Blandford, R. D., \& Rees, M. J. 1974, MNRAS, 169, 395

Bogers, W. J., Hes, R., Barthel, P. D., \& Zensus, J. A. 1994, A\&AS, 105, 91 Bridle, A. H., Hough, D. H., Lonsdale, C. J., Burns, J. O., \& Laing, R. A. 1994, AJ, 108, 766

Fanaroff, B. L., \& Riley, J. M. 1974, MNRAS, 167, 31P

Fernini, I. 2007, AJ, 134, 158

Fernini, I., Burns, J. O., \& Perley, R. A. 1997, AJ, 114, 2292

Garrington, S. T., Leahy, J. P., Conway, R. G., \& Laing, R. A. 1988, Nature, 331, 147

Garrington, S. T., Conway, R. G., \& Leahy, J. P. 1991, MNRAS, 250, 171

Gawroński, M. P., Marecki, A., Kunert-Bajraszewska, M., \& Kus, A. J. 2006, A\&A, 447, 63

Gilbert, G. M., Riley, J. M., Hardcastle, M. J., et al. 2004, MNRAS, 351, 845 (G04)

Gopal-Krishna, \& Wiita, P. J. 2000, A\&A, 363, 507

Gopal-Krishna, Wiita, P. J., \& Hooda, J. S. 1996, A\&A, 316, L13

Hardcastle, M. J., Alexander, P., Pooley, G. G., \& Riley, J. M. 1997, MNRAS, 288,859

Harvanek, M., \& Hardcastle, M. J. 1998, ApJS, 119, 25

Jorstad, S. G., \& Marscher, A. P. 2008, Extragalactic Jets: Theory and Observation from Radio to Gamma Ray, ASP Conf. Ser., 386, 219

Kaiser, C. R., Schoenmakers, A. P., \& Röttgering, H. J. A. 2000, MNRAS, 315, 381

Kharb, P., O’Dea, C. P., Baum, S. A., et al. 2008, ApJS, 174, 74

Konar, C., Hardcastle, M. J., Jamrozy, M., Croston, J. H., \& Nandi, S. 2012, MNRAS, 424, 1061

Laing, R. A. 1988, Nature, 331, 149

Law-Green, J. D. B., Leahy, J. P., Alexander, P., et al. 1995, MNRAS, 274, 939

Longair, M. S., \& Riley, J. M. 1979, MNRAS, 188, 625

Lonsdale, C. J., \& Morison, I. 1983, MNRAS, 203, 833

Lonsdale, C. J., Barthel, P. D., \& Miley, G. K. 1993, ApJS, 87, 63

Marecki, A. 2012, A\&A, 544, L2 (Paper I)

Marecki, A., \& Swoboda, B. 2011, A\&A, 525, A6

Neff, S. G., Roberts, L., \& Hutchings, J. B. 1995, ApJS, 99, 349

Pirya, A., Nandi, S., Saikia, D. J., \& Singh, M. 2011, BASI, 39, 547

Price, R., Gower, A. C., Hutchings, J. B., et al. 1993, ApJS, 86, 365

Reid, A., Shone, D. L., Akujor, C. E., et al. 1995, A\&AS, 110, 213

Reid, R. I., Kronberg, P. P., \& Perley, R. A. 1999, ApJS, 124, 285

Riley, J. M., Rawlings, S., McMahon, R. G., et al. 1999, MNRAS, 307, 293

Saikia, D. J., \& Jamrozy, M. 2009, BASI, 37, 63

Schoenmakers, A. P., de Bruyn, A. G., Röttgering, H. J. A., \& van der Laan, H. 2000, MNRAS, 315, 395 